\title{
Characterizing carbonyl compounds and their sources in Fuzhou ambient air, southeast of China
}

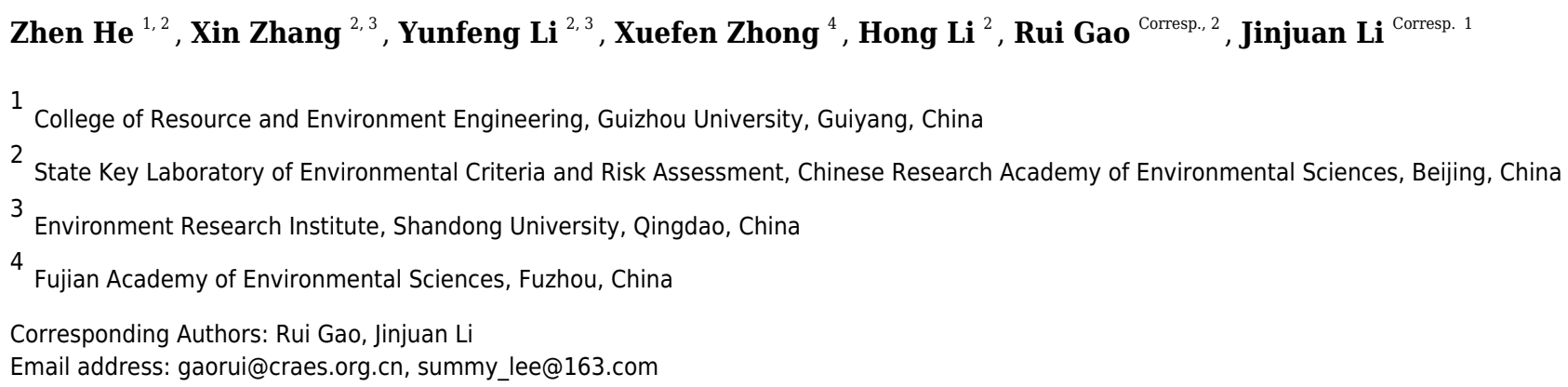

In recent years, ozone $\left(\mathrm{O}_{3}\right)$ concentrations in the southeastern coastal areas of China have shown a gradual upward trend. As precursors and intermediates in the formation of $\mathrm{O}_{3}$, carbonyl compounds play key roles in the atmospheric photochemical oxidation cycle. To explore the main pollution characteristics of carbonyl compounds in a typical coastal city in southeast China, ambient samples were collected in Fuzhou (The provincial capital of Fujian province, located on the southeast coast of China) and analyzed using highperformance liquid chromatography with ultraviolet detection. The study was continuously carried out at an urban site (Jinjishan) and a suburban site (Gushan) in Fuzhou from May 8 to 20,2018 . The total concentration of 16 carbonyl compounds at the urban site was 15.45 $\pm 11.18 \mathrm{ppbv}$, and the total concentration at the suburban site was $17.57 \pm 12.77 \mathrm{ppbv}$. Formaldehyde ( $\mathrm{HCHO})$, acetaldehyde, and acetone were the main species detected in the samples, and acetone had the highest concentration among the species detected. The suburban site had a higher formaldehyde/acetaldehyde ratio and lower acetaldehyde/propionaldehyde ratio than the urban site, implying that biogenic sources potentially contributed to the carbonyl compound concentrations at the suburban site. The results of an observation-based model showed that anthropogenic hydrocarbons promoted $\mathrm{HCHO}$ production on May 17 at the urban site. Compared to biogenic emissions, anthropogenic activity is a more important source of carbonyl compounds. 


\section{Characterizing carbonyl compounds and their sources}

\section{2 in Fuzhou ambient air, southeast of China}

3 Zhen $\mathrm{He}^{1,2}$, Xin Zhang ${ }^{2,3}$, Yunfeng $\mathrm{Li}^{2,3}$, Xuefen Zhong ${ }^{4}$, Hong Li², Rui Gao ${ }^{2}$, Jinjuan Li

$4{ }^{1}$ College of Resource and Environment Engineering, Guizhou University, Guiyang, China

52 State Key Laboratory of Environmental Criteria and Risk Assessment, Chinese Research

6 Academy of Environmental Sciences, Beijing, China

$7 \quad{ }^{3}$ Environment Research Institute, Shandong University, Qingdao, China

$8{ }^{4}$ Fujian Academy of Environmental Sciences, Fuzhou, China

Corresponding Author:

11 Rui Gao

12 No. 8 Dayangfang Street, Chaoyang District, Beijing, 100055, China

13 Email address: gaorui@craes.org.cn

14

Jinjuan Li

16 No. 2708, Huaxi Dadao Street, Guiyang, 550025, China

17 Email address: summy_lee@163.com

18

19

20

21

22

23

24

25 


\section{Characterizing carbonyl compounds and their sources \\ 28}

29

30

31

32

33

34

35

36

37

38

39

40

41

42

43

44

45

46

47

48

49

50

51

52

53

54

55

56

57

58

59

60

61

62

63

64

65

Zhen $\mathrm{He}^{1,2}$, Xin Zhang ${ }^{2,3}$, Y unfeng $\mathrm{Li}^{2,3}$, Xuefen Zhong ${ }^{4}$, Hong $\mathrm{Li}^{2}$, Rui Gao ${ }^{2}$, Jinjuan $\mathrm{Li}^{1}$

${ }^{1}$ College of Resource and Environment Engineering, Guizhou University, Guiyang, China

${ }^{2}$ State Key Laboratory of Environmental Criteria and Risk Assessment, Chinese Research Academy of Environmental Sciences, Beijing, China

${ }^{3}$ Environment Research Institute, Shandong University, Qingdao, China

${ }^{4}$ Fujian Academy of Environmental Sciences, Fuzhou, China

Corresponding Author:

Rui $\mathrm{Gao}^{2}$

No. 8 Dayangfang Street, Chaoyang District, Beijing, 100055, China

Email address: gaorui@craes.org.cn

Jinjuan $\mathrm{Li}^{1}$

No. 2708, Huaxi Dadao Street, Guiyang, Guizhou, 550025, China

Email address: summy lee@,163.com

\section{Abstract}

In recent years, ozone $\left(\mathrm{O}_{3}\right)$ concentrations in the southeastern coastal areas of China have shown a gradual upward trend. As precursors and intermediates in the formation of $\mathrm{O}_{3}$, carbonyl compounds play key roles in the atmospheric photochemical oxidation cycle. To explore the main pollution characteristics of carbonyl compounds in a typical coastal city in southeast China, ambient samples were collected in Fuzhou (The provincial capital of Fujian province, located on the southeast coast of China) and analyzed using high-performance liquid chromatography with ultraviolet detection. The study was continuously carried out at an urban site (Jinjishan) and a suburban site (Gushan) in Fuzhou from May 8 to 20, 2018. The total concentration of 16 carbonyl compounds at the urban site was $15.45 \pm 11.18 \mathrm{ppbv}$, and the total concentration at the suburban site was $17.57 \pm 12.77 \mathrm{ppbv}$. Formaldehyde ( $\mathrm{HCHO}$ ), acetaldehyde, and acetone were the main species detected in the samples, and acetone had the highest concentration among the species detected. The suburban site had a higher formaldehyde/acetaldehyde ratio and lower acetaldehyde/propionaldehyde ratio than the urban site, implying that biogenic sources potentially contributed to the carbonyl compound concentrations at the suburban site. The results of an observation-based model showed that anthropogenic hydrocarbons promoted $\mathrm{HCHO}$ production on May 17 at the urban site. Compared to biogenic emissions, anthropogenic activity is a more important source of carbonyl compounds. 


\section{Introduction}

67 Carbonyl compounds are precursors of $\mathrm{O}_{3}$ and secondary organic aerosols, and play key roles in

68

69

70

71

72

73

74

75

76

77

78

79

80

81

82

83

84

85

86

87

88

89

90

91

92

93

94

95

96

97

98

99

100

101

102

103

104

105 the atmospheric photochemical oxidation cycle. The impact of carbonyl compounds on $\mathrm{O}_{3}$ pollution has become a hot topic in the field of atmospheric chemistry research (Finlayson-Pitts \& Pitts, 1997; Zhu et al., 2019). These species can control the generation rates and efficiency of hydroxyl $\left(\mathrm{HO}_{2}\right)$ and peroxyalkyl $\left(\mathrm{RO}_{2}\right)$ groups in the atmosphere through their photolysis and reactions with $\mathrm{OH}$ radicals. Carbonyls are also important intermediates in the photo-oxidation of volatile organic compounds (VOCs) and important precursors of peroxyacetyl nitrate (PAN) and organic acids, which means they have an important influence on the potential effect of atmospheric oxidation on $\mathrm{O}_{3}$ pollution.

Carbonyl compounds undergo oxidation reactions with atmospheric oxidants to affect the equilibrium relationship in the photochemical oxidant cycle. At the same time, they are oxidized to form less volatile organic compounds, which gradually enter the particulate phase through condensation and adsorption processes and then form secondary organic aerosols (Crutzen and Andreae, 1990, Brey and Fischer, 2016). Carbonyls also have a direct negative impact on human health. Most aldehydes and ketones are strongly irritating, and can cause respiratory infections, sensitization, carcinogenesis, and mutation. Most of the unhealthy symptoms induced by carbonyl compounds are irritation to the eyes and lungs (WHO, 2000). Among them, the International Agency for Research on Cancer classifies formaldehyde as the first category of human carcinogens (IARC, 2006), which can cause nasopharyngeal cancer (IARC, 2004), and may also be related to leukemia (Zhang et al., 2009).

Carbonyl compounds are abundant components in ambient air. They are mainly formed by the oxidation of biogenic and anthropogenic hydrocarbons (Zhu et al., 2019), and are also directly emitted from natural and anthropogenic sources (Singh et al., 1995; Wu \& Wang, 2015; Xiang et al., 2017; Zarzana et al., 2017). Generally, anthropogenic sources are significant in urban areas. Biological sources of carbonyl compounds are also considered important.

In China, the concentrations of carbonyl compounds in the atmosphere are usually high. For example, the atmospheric concentrations of carbonyl compounds measured in Beijing showed that the environmental level of carbonyl compounds is 3-5 times of that in Hong Kong (Pang \& $M u$, 2006). The concentration of HCHO in Guangzhou is 3 times that in Japan and 2 times that in Hong Kong (Feng et al., 2005; Sin et al., 2001; Tago et al., 2005). The high concentrations of carbonyl compounds may be important contributors to serious atmospheric photochemical pollution in Chinese cities.

Fuzhou is a city with relatively good air quality in China, ranking 6th out of 168 key cities in China for ambient air quality in 2019. However, ozone concentrations show an increasing trend year by year. Fuzhou's current $\mathrm{O}_{3}$ pollution situation is likely to be a brand-new challenge for China to

Peer] reviewing PDF | (2020:06:50164:1:0:NEW 9 Sep 2020) 
106 face after $\mathrm{PM}_{2.5}$ pollution is controlled. Therefore, the situation in Fuzhou deserves attention. The
107 carbonyl compounds are important precursors of $\mathrm{O}_{3}$, and the study of its characteristics is of great

107

108

109

110

111

112

113

114

115

116

117

118

119

120

121

122

123

124

125

126

127

128

129

130

131

132

133

134

135

136

137

138

139

140

141

142

143

144

145 significance to the control of $\mathrm{O}_{3}$ pollution. As important precursors of $\mathrm{O}_{3}$, VOCs and $\mathrm{NOx}$ have attracted wide attention (Hong et al., 2019; Wang et al., 2017). However, most research to date has focused on non-methane hydrocarbons and carbonyl compounds have received limited attention. This research focuses on Fuzhou carbonyl compounds to explore their pollution characteristics and their relationship with ozone concentration. In this study, carbonyl compounds in ambient air were investigated using offline 2,4-dinitrophenylhydrazine (DNPH) cartridge sampling with high-performance liquid chromatography (HPLC) analysis. Fuzhou is in the western part of the Taiwan Strait and is subject to weaker winds during the monsoon exchange period in May and is less affected by regional transmission. Sixteen carbonyl compounds were analyzed in samples collected from May 8 to 20, 2018 in Fuzhou.

\section{Materials \& Methods Sampling sites}

Two observation sites were chosen on the rooftop of the Jinjishan Environmental Protection Building (Jijishan site: JJS) and in the Gushan Scenic Area (Gushan site: GSS) in the suburbs of Fuzhou, China (Fig. 1).

Fuzhou is located on the southeast coast of China. The JJS site was located $30 \mathrm{~m}$ above the ground at the foot of the southwest Jinjishan mountain in the Jin'an district, Fuzhou. The Jin'an River was located to the west of this site and Jinjishan Park to the northeast. The site was surrounded by residential areas and was considered a typical urban site with no pollution sources. The GSS site was located on the top of the Songtao Building of the Gushan Scenic Area Management Committee. This site was located to the south of the east side of Fuzhou city and north of the Minjiang River. The GSS was selected by China National Environmental Monitoring Center (CNEMC) as a suburban background site as it was surrounded by green trees and had no pollution sources. This site was located about $8 \mathrm{~km}$ away from the JJS site.

\section{Carbonyls sampling and analysis}

The sampling and analysis procedures used were based on the Environmental Protection Agency method TO-11A (US EPA, 1999). Ambient carbonyls were collected on silica cartridges coated with acidified DNPH (IC-DN3501, Agela Technologies, China). The sampling flow rate was 0.8 $\mathrm{L} / \mathrm{min}$. The sampling pumps in this study are all vacuum pumps with a rated voltage of $24 \mathrm{v}$, and soap film flowmeters are used for flow calibration before sampling (Zhang et al. 2018). Samples were collected at a $2 \mathrm{~h}$ intervals beginning at 00:00 (UTC+8) from May 8 to 20, 2018. The sampling duration was $2 \mathrm{~h}$ with 12 atmospheric samples collected each day: 00:00-02:00; 02:0004:00; 04:00-06:00; 06:00-08:00; 08:00-10:00; 10:00-12:00; 12:00-14:00; 14:00-16:00; 16:00$18: 00 ; 18: 00-20: 00 ; 20: 00-22: 00 ; 22: 00-00: 00$. A total of 156 carbonyl compound samples were collected at each site. $\mathrm{An}_{3}$ scrubber loaded with potassium iodide (KI 140, Agela Technologies,

Peer] reviewing PDF | (2020:06:50164:1:0:NEW 9 Sep 2020) 
146 China) was installed in front of the cartridge to eliminate $\mathrm{O}_{3}$. Two cartridges in series were sampled 147 to evaluate the collection efficiency before application to the first sample collection in the field. 148 Over $98 \%$ of carbonyls were detected in the first cartridge. After sampling, the cartridges were 149 sealed with Teflon caps immediately, placed in an aluminum foil bag to protect them from light, 150 and stored in a refrigerator $\left(<4^{\circ} \mathrm{C}\right)$. Throughout the sampling process, blank samples were 151 collected at each location. Blank samples were collected by passive sampling, which involved 152 opening a sealed cartridge and placing it in the ambient air at the same time as a cartridge was used 153 for active air sampling. Six cartridges were collected every 12 hours, and the concentration of 154 blank samples with no sampling for 12 hours had been deducted from the concentration of each 155 sample. Two filed blank samples were collected both before and after the sampling process. At the 156 end of the sample collection period, all the samples were transported to the Laboratory of Atmospheric Photochemical Simulation at the Chinese Research Academy of Environmental Sciences (Beijing, China) in a heat-resistant incubator maintained at $0^{\circ} \mathrm{C}$ and then analyzed within 1 month.

160

In the sampling cartridges, the ambient carbonyls reacted with DNPH to form stable hydrazone derivatives. These derivatives were eluted slowly from the cartridges into a volumetric flask using $5 \mathrm{~mL}$ of acetonitrile (HPLC grade, J.T. Baker, USA). No DNPH or derivatives remained in the cartridges after this elution. Because we several eluted cartridges were chosen to be eluted with 5 $\mathrm{ml}$ of acetonitrile again, and no residual carbonyl compounds in the second eluted solutions were detected. This shows that $5 \mathrm{ml}$ of acetonitrile is enough to elute all the carbonyl compounds in the cartridges. The extracts were then analyzed by HPLC (LC20A, Shimadzu, Japan) with UV-Vis detection at $360 \mathrm{~nm}$. The separation column was a Inertsil ODS-P reversed-phase column $(250 \times$ $4.6 \mathrm{~mm}$ i.d., $5 \mu \mathrm{m}$ particle size; Shimadzu, Japan) at $40^{\circ} \mathrm{C}$. The mobile phase consisted of acetonitrile and water and the following gradient elution was used: 0-20 min, $60 \%$ acetonitrile; $20-30 \mathrm{~min}, 60 \%$ to $100 \%$ acetonitrile; $30-32 \mathrm{~min}, 100 \%$ to $60 \%$ acetonitrile; and $32-40 \mathrm{~min}, 60 \%$ acetonitrile. The total flow rate was $1.0 \mathrm{~mL} / \mathrm{min}$ and the injection volume was $20 \mu \mathrm{L}$. A mixed calibration standard of 15 DNPH-carbonyl derivatives (Supelco, Bellefonte, PA), which contained HCHO, acetaldehyde, acrolein, acetone, propionaldehyde, crotonaldehyde, butyraldehyde, benzaldehyde, isovaleraldehyde, valeraldehyde, o-tolualdehyde, m-tolualdehyde, p-tolualdehyde, hexaldehyde, and 2,5-dimethylbenzaldehyde, and a calibration standard of methacrylaldehyde (MACR) were diluted to $0.03,0.06,0.09,0.15,0.24,0.30$, and $0.45 \mu \mathrm{g} / \mathrm{mL}$. Details for the detection of the 16 carbonyl compounds are given in Table 1 .

179

\section{Other measurements}

The $\mathrm{O}_{3}$ concentrations were measured by a UV photometric $\mathrm{O}_{3}$ analyzer (Model 49i, Thermo Fisher Scientific, USA). The $\mathrm{NO}$ and $\mathrm{NO}_{2}$ concentrations were measured by a chemiluminescence instrument (Model 42i, Thermo Fisher Scientific) coupled with a molybdenum oxide catalytic converter. The $\mathrm{SO}_{2}$ and $\mathrm{CO}$ concentrations were measured by a pulsed fluorescence analyzer (Model 43i, Thermo Fisher Scientific) and an infrared absorption analyzer (Model 48i, Thermo 
186 Fisher Scientific), respectively. The $\mathrm{PM}_{2.5}$ concentrations were measured by a multiangle

187

188

189

190

191

192

193

194

195

196

197

198

199

200

201

202

203

204

205

206

207

208

209

210

211

212

213

214

215

216

217

218

219

220

221

222

223

224

225

absorption photometer (Model 5012, Thermo Fisher Scientific). The pollutants data (including $\mathrm{O}_{3}$, $\mathrm{CO}, \mathrm{NO}_{2}, \mathrm{SO}_{2}, \mathrm{PM}_{2.5}$ ) came from the National Environmental Monitoring Station in Fuzhou. All instruments in these sites are maintained by a professional service company every day and are turned on during the carbonyl compound sampling period.

The VOCs concentrations were determined on an $\mathrm{O}_{3}$ pollution day (May 17) at the JJS site. Four samples were collected in 3.2-L stainless steel canisters at 8:00, 14:00, 18:00, and 21:00 local time. Fifty-seven VOCs species of Photochemical Assessment Monitoring Stations (PAMS) were identified using a gas chromatograph equipped with a flame ionization detector and a mass spectrometry detector (EPA/600-R-98/161, Technical Assistance Document for Sampling and Analysis of Ozone Precursors) at the same laboratory in Chinese Research Academy of Environmental Sciences. Meteorological data for the temperature, relative humidity, wind speed, wind direction, and pressure were obtained from the National Climate Data Center of the National Oceanic and Atmospheric Administration (National Climatic Data Center).

\section{Observation-based model}

For quantification of the in situ photochemical production and sensitivity analysis of carbonyl compounds, an observation-based model (OBM) was utilized in this study. This model has been successfully applied in previous studies (He et al., 2019; Mellouki et al., 2015; Xue et al., 2014; Yang et al., 2018; Zhang et al., 2019). Briefly, it is built on the Master Chemical Mechanism, which is a near-explicit mechanism describing the oxidation of 143 primary VOCs together with the latest IUPAC inorganic nomenclature (Jenkin et al., 2003; Saunders et al., 2003). In this study, the model was updated to the newest version of the Master Chemical Mechanism (MCM website). In the calculations, the observed concentrations of $\mathrm{O}_{3}, \mathrm{NO}, \mathrm{NO}_{2}, \mathrm{SO}_{2}, \mathrm{CO}$, and VOCs, and the temperature, relative humidity, and pressure were interpolated to a time resolution of $1 \mathrm{~h}$ and processed as the model input data sets. We only analyzed the case at JJS station on May 18, 2018 because of the VOCs data limited.

\section{Results}

\section{Concentration level}

Fig. 2 shows the measured time series of major carbonyl compounds, $\mathrm{O}_{3}, \mathrm{NO}_{2}, \mathrm{SO}_{2}, \mathrm{CO}, \mathrm{PM}_{2.5}$, and meteorological parameters at the JJS and GSS sites in Fuzhou from May 8 to 20, 2018. The average concentrations of $\mathrm{O}_{3}, \mathrm{NO}_{2}, \mathrm{SO}_{2}, \mathrm{CO}$, and $\mathrm{PM}_{2.5}$ during the observation period at the GSS site were $48.29 \mathrm{ppbv}, 6.99 \mathrm{ppbv}, 2.67 \mathrm{ppbv}, 0.47 \mathrm{ppmv}$, and $29.40 \mu \mathrm{g} / \mathrm{m}^{3}$, respectively. At the JJS site, the corresponding average concentrations were $40.44 \mathrm{ppbv}, 14.18 \mathrm{ppbv}, 1.63 \mathrm{ppbv}, 0.62$ ppmv, and $31.06 \mu \mathrm{g} / \mathrm{m}^{3}$ (Table 2).

During the observation period, the concentrations of carbonyl compounds at the JJS and GSS sites were relatively low from May 8 to 11, and the. This may have been caused by low temperatures

PeerJ reviewing PDF | (2020:06:50164:1:0:NEW 9 Sep 2020) 
226 (average temperature was $21.3{ }^{\circ} \mathrm{C}$ ) that were not conducive to the formation of carbonyl 227 compounds or strong southwesterly winds that facilitated dispersion of the pollutants. From May 22812 to 18 , the carbonyl compound concentrations at the two sites greatly increased, which may have 229 been caused by high temperatures (average temperature was $27.1{ }^{\circ} \mathrm{C}$ ) and strong easterly winds 230 facilitating the formation and accumulation of pollutants. On May 19 and 20, the temperature 231 dropped and the wind direction changed, which resulted in decreases in the concentrations of carbonyl compounds at the two sites. The $\mathrm{O}_{3}$ concentration changes were consistent with those for

233

234

235

236

237

238

239

240

241

242

243

244

245

246

247

248

249

250

251

252

253

254

255

256

257

258

259

260

261

262

263

264

265 the carbonyl compounds during the observation period. The $\mathrm{O}_{3}$ concentration remained at a low level from May 8 to 11, greatly increased from May 12 to 18, and slightly decreased on May 19 and 20. In addition, from May 12 to 20, the GSS site showed large diurnal variations in the concentrations of $\mathrm{O}_{3}$ and the carbonyl compounds, and these concentrations peaked at almost the same time (around noon), which indicated that photochemical reactions were the main contributor to $\mathrm{O}_{3}$ and carbonyl compound formation during the day at the GSS site. A similar situation was also observed at the JJS site. However, from May 13 to 19 at the JJS site, no obvious daily trends were observed in the carbonyl compound concentrations. These results suggested that anthropogenic sources around the site had considerable contributions in addition to the contribution of photochemical reactions during the day. These observations indicate that the photochemical pollution phenomena occur in Fuzhou.

The overall trends for the carbonyl compounds at the two sites were similar, with a peak in the middle of the day, but the carbonyl compound composition varied greatly with time.

Among the carbonyl compounds detected at the GSS site from May 8 to 11, the dominant carbonyl compound was acetaldehyde, followed by HCHO. From May 12 to 13, the concentration of acetaldehyde increased sharply, and the main reason for this was the overall increase in the concentration of carbonyl compounds. In addition, the concentrations of other carbonyl compounds also increased. From May 14 to 17, the concentration of acetaldehyde decreased and that of acetone increased rapidly. The increasing trend of acetone is due to the conversion of acetaldehyde and the gradual accumulation of acetone. In addition, changes in wind direction and the influence of other man-made pollution sources may be the cause of this phenomenon. The concentration of acetone was much higher than that of any other species. During the observation period, the concentrations of the species varied greatly. The daily concentration changes were basically the same, indicating a stable source of the carbonyl compounds. From May 18 to 20, the total concentration of carbonyl compounds declined as the acetone concentration decreased.

From May 8 to 14, the JJS site was similar to the GSS site and the carbonyl compound concentration was relatively low. Starting on May 14, the concentration of acetaldehyde increased greatly and the concentration of acetone increased slightly, which was the main reason for the large increase in the carbonyl compound concentration. From May 15 to 18 , the concentration of acetone increased rapidly and it became the main species, followed by acetaldehyde and $\mathrm{HCHO}$, and the concentrations of the compounds continued to increase. From May 18 to 20, the concentration of

Peer) reviewing PDF | (2020:06:50164:1:0:NEW 9 Sep 2020) 
266

267

268

269

270

271

272

273

274

275

276

277

278

279

280

281

282

283

284

285

286

287

288

289

290

291

292

293

294

295

296

297

298

299

300

301

302

303

304

305

acetone began to decrease relative to the previous period (May 15 to 18) but the concentration remained high and it was still the dominant species, followed by acetaldehyde and HCHO.

Table 3 shows the average concentrations and ranges of the 16 carbonyl compounds detected at the GSS and JJS sites in Fuzhou. The total average concentration ( \pm standard deviation) of carbonyl compounds at the GSS site was $17.57 \pm 12.77 \mathrm{ppbv}$ and the range was $1.11-53.22 \mathrm{ppbv}$. The total average concentration of carbonyl compounds at the JJS site was slightly lower than that at the GSS site, which was $15.45 \pm 11.18 \mathrm{ppbv}$ with a range of $0.04-46.83 \mathrm{ppbv}$.

\section{Diurnal variations in the ambient Carbonyl compounds}

Fig. 3 shows diurnal variation of the total carbonyl compound and $\mathrm{O}_{3}$ concentrations at the GSS and JJS sites from May 18 to 20, 2018. Both sites showed diurnal variation. It is well-known that atmospheric photochemical reactions are one of the important production routes of carbonyl compounds in ambient air and an important method of $\mathrm{O}_{3}$ generation. The intensity of solar radiation directly affects reaction rates in atmospheric photochemistry. Therefore, the intensity of solar radiation has an important influence on the concentrations of carbonyl compounds and $\mathrm{O}_{3}$. At the same time, anthropogenic emissions are also important and can affect the concentrations of carbonyl compounds, which directly affect the diurnal variation of carbonyl compounds.

Diurnal variation of the total carbonyl compounds at the GSS site showed a distinct single peak during the observation period. Overall, the total concentration of carbonyl compounds was much higher during the day than at night, with concentrations beginning to increase around 05:00 and peaking at around 12:00-14:00 when solar radiation was the strongest. As solar radiation decreased, the concentration decreased rapidly from 14:00 to 19:00 and stabilized after 19:00. From 19:00 to 05:00 the next day, the concentrations of the carbonyl compounds did not vary much and were maintained at low levels relative to those observed during the day. During the observation period, the daily change in the $\mathrm{O}_{3}$ concentration at the GSS site also showed a single peak, and the trend was similar to that observed for the carbonyl compounds.

Diurnal variation at the JJS site was different to that at the GSS site. Generally, variation in the concentrations of carbonyl compounds at the JJS site showed multiple peaks. Similar to the GSS site, the JJS site had higher concentrations of carbonyl compounds during the day than at night; however, the JJS site had much higher concentrations at night (19:00-07:00) than the GSS site, indicating a persistent source of pollution. During the day, the carbonyl compounds showed a peak at 07:00, concentrations then rose from 05:00 to 07:00 before decreasing slightly from 07:00 to 09:00. As solar radiation increased, the concentrations of the carbonyl compounds increased again after 09:00. Between 13:00 and 15:00, the atmospheric photochemical reaction rate reached its daytime peak, and the highest carbonyl compound concentrations were reached at 15:00. From 15:00 to 19:00, as solar radiation decreased, the concentrations of the carbonyl compounds gradually decreased, and between 19:00 to 23:00, a late peak for the carbonyl compounds was

Peer) reviewing PDF | (2020:06:50164:1:0:NEW 9 Sep 2020) 
306

307

308

309

310

311

312

313

314

315

316

317

318

319

320

321

322

323

324

325

326

327

328

329

330

331

332

333

334

335

336

337

338

339

340

341

342

343

344

345

reached and then the concentrations began to decrease. The diurnal variation of $\mathrm{O}_{3}$ at the JJS site basically showed a single peak that was consistent with the variation observed for the carbonyl compounds from 9:00 to 19:00. This indicated that the concentrations of the carbonyl compounds were mainly affected by atmospheric photochemical reactions during this period.

Fig. 4 shows diurnal variation of the main carbonyl compounds at the two sites from May 8 to 20, 2018. The overall diurnal variation trend for these compounds was similar to that of the total carbonyl compounds. The concentrations of acetaldehyde and acetone at the GSS site were much higher than those of other species, indicating that this was the main species that affected diurnal variation. The diurnal variation of acetaldehyde at the JJS site was consistent with the change in the total carbonyl compounds, indicating that it was one of the main species that affected the total diurnal variation of carbonyl compounds at the JJS site. In addition, the high concentration of acetone also affected the diurnal variation trend of the total carbonyl compounds at the JJS site to a certain extent.

\section{Discussion}

\section{Comparison of urban and suburban stations}

The carbonyl compounds at the GSS site, which had less human activity than the JJS site, mainly arise from secondary generation via photochemical reactions. By contrast, at the JJS site, in addition to secondary generation, human activity in the surrounding area has a high contribution to the carbonyl compound concentrations.

The JJS site was located in an urban area, the pollution sources were more complex than at the GSS site, and the carbonyl compound sources may also be more complex. Although the GSS site was located in a forested area with a single main source of carbonyl compounds, it was close to the JJS site and could be affected pollutants transported from other areas. This may be the main reason why the concentration of carbonyl compounds at the GSS site was slightly higher than that at the JJS site.

Fig. 5 shows the average contributions of different carbonyl compounds at the two sites. Acetone was the most abundant carbonyl compound at the GSS and JJS sites, which could be attributed to its chemical stability and long atmospheric lifetime. Acetone released from various sources accumulates in the atmosphere (Atkinson, 2000; Dai et al., 2012), and the most important sources are natural sources (Janson et al., 2001), with pine trees accounting for a higher share of emissions. Among the anthropogenic sources, motor vehicle exhaust emissions are more significant ( $\mathrm{Ho} \mathrm{KF}$ et al., 2015). The average concentration of acetone at the GSS site was $7.45 \pm 8.13 \mathrm{ppbv}$ (Table 3 ), accounting for $42.26 \%$ of the total concentration of the 16 carbonyl compounds. The average acetone concentration at the JJS site $(6.82 \pm 8.11 \mathrm{ppbv})$ (Table 3$)$ was slightly lower than that at the GSS site, accounting for $44.14 \%$ of the total concentration of the 16 carbonyl compounds. These results were comparable to the acetone concentrations measured by Yang et al. in Beijing 
346 (Yang et al., 2018). They were also similar to the concentrations measured by Lü et al. in 347 Guangzhou, where acetone was one of the species with the highest concentrations (Lü et al., 2010).

348 The compound with the second highest concentration at the GSS site was acetaldehyde $(4.41 \pm$ $3494.36 \mathrm{ppbv}, 25.01 \%$ of the total) (Table 3$)$, and the third was HCHO $(2.54 \pm 2.09 \mathrm{ppbv}, 14.41 \%$ of 350 the total) (Table 3). Similar to the GSS site, the species with second highest concentration at the

351

352

353

354

355

356

357

358

359

360

361

362

363

364

365

366

367

368

369

370

371

372

373

374

375

376

377

378

379

380

381

382

383

384

385 JJS site was acetaldehyde $(4.84 \pm 3.63 \mathrm{ppbv}, 31.35 \%$ of the total), and the third was HCHO oxidation ( $1.64 \pm 0.75 \mathrm{ppbv}, 10.64 \%$ of the total) (Table 3 ). Higher concentration of MACR could be caused by the abundant vegetation at the GSS site, which would produce more MACR than at the JJS site (Duane et al., 2002; Riemer et al., 1998).

\section{Diurnal variation analysis}

The concentration of carbonyl compounds at GSS sites has an obvious single-peak diurnal variation trend, which is basically consistent with the change of solar radiation intensity during a day, indicating that the concentration of carbonyl compounds is mainly caused by atmospheric photochemical reactions. However, there is no significant diurnal variation in the concentration of carbonyl compounds from the whole-time variation at the JSS site, indicating that the concentration of carbonyl compounds is not only influenced by atmospheric photochemical reactions, but also by certain anthropogenic factors.

The highest concentrations of acetone could be observed at the two sites at any time. Atmospheric oxidation affects the atmospheric lifetime of carbonyl compounds. The photolysis reactions of $\mathrm{HCHO}$ and acetaldehyde take about $4 \mathrm{~h}$ and 6 days, respectively, and their lifetimes initiated by $\mathrm{OH}$ radicals are about 1.2 days (HCHO) and $8.8 \mathrm{~h}$ (acetaldehyde) (Dai et al., 2012). Acetone is more stable and has a longer lifetime (60 days uptake by photolysis and 53 days uptake by reaction with $\mathrm{OH}$ radicals) than those of $\mathrm{HCHO}$ and acetaldehyde. Therefore, acetone accumulates in the atmosphere easily and its concentration is higher than those of other carbonyl compounds such as HCHO and acetaldehyde (Atkinson, 2000; Possanzini et al., 2007). This further explains why the acetone concentration is much higher than other carbonyl compounds.

\section{Source apportionment of carbonyl compounds using ratio methods}

The sources of carbonyl compounds can be roughly determined from the ratio of the concentrations of atmospheric formaldehyde/acetaldehyde (F/A) to that of acetaldehyde/propionaldehyde (A/P) (Shepson et al., 1991). Fig. 6 and Fig. 7 show statistical analysis of the F/A and A/P ratios during the day and night at the GSS and the JJS sites from May 8 to 21 .

The F/A value in the atmosphere is generally between 1-10. Generally, the amount of formaldehyde produced by biogenic-derived hydrocarbons through atmospheric photochemical reactions is higher than the amount of acetaldehyde. Therefore, the F/A ratio is higher in areas with high forest or vegetation coverage than in urban areas (Possanzini et al., 1996; Shepson et al., 1991). Overall, the F/A value at the GSS site (daytime: 0.19-1.38, nighttime: 0.10-1.42) first 
386

387

388

389

390

391

392

393

394

395

396

397

398

399

400

401

402

403

404

405

406

407

408

409

410

411

412

413

414

415

416

417

418

419

420

421

422

423

424

425

decreased and then increased during the observation period. The GSS site was located in a forested area and there was one main source of carbonyl compounds. However, the F/A values at the GSS site were much lower than those in typical forested areas, which may be related to the regional transportation of pollutants. During the observation period, the F/A value at the JJS site (daytime: 0.18-0.69, nighttime: $0.10-1.43$ ) also first decreased and then increased.

Overall, the F/A value at the GSS site was slightly higher than that at the JJS site during the observation period. This may be because the vegetation coverage at the GSS site was higher than that at the JJS site as the GSS site was in a forested area. Therefore, the F/A value at the GSS site was higher than that at the urban JJS site. However, because the GSS site was located close to an urban area, the F/A value was lower than that in typical forested area because of the impact of pollutant transportation. The F/A values were slightly higher at night than during the day at both sites, which could be attributed to the fact that formaldehyde and acetaldehyde were removed at night mainly by reactions with $\mathrm{NO}_{3}$ radicals. Acetaldehyde reacted at a higher rate than formaldehyde at night. From May 12 to 13, a sudden increase in the GSS acetaldehyde concentration led to a decrease in its F/A value to slightly lower than that at the JJS site.

Generally, the A/P ratio can be used to indicate the presence of man-made pollutants because propionaldehyde is considered only to be associated with anthropogenic sources (Shepson et al., 1991). Therefore, the lower the A/P value, the greater the influence of anthropogenic sources. The $\mathrm{A} / \mathrm{P}$ at the GSS website was between 2.22-23.64 during the day and 2.20-28.77 at night, while at the JJS site, the A/P was between 2.52-31.62 during the day and 2.99-39.09 at night. Propionaldehyde is mainly related to anthropogenic emissions, and acetaldehyde may come from secondary generation or primary emission. The A/P value at the JJS site was higher than the A/P value at the GSS site. From the A/P ratio, the GSS site seems to be more severely affected by manmade sources, which may be due to the GSS site producing more acetaldehyde. And the GSS site was affected by pollutant transportation from the source area (Table 3), and the concentration of propionaldehyde was slightly higher than that at the JJS site.

The ratio of the concentrations of atmospheric formaldehyde/acetaldehyde (F/A) and acetaldehyde/propionaldehyde (A/P) can be analyzed the relative contribution of anthropogenic and biogenic sources. However, there were some arguments that the ratios of F/A and A/P often have large variations due to different sources of pollution and meteorological conditions (Grosjean, 1992; Ho et al., 2015), thus we should use it with caution. Furthermore, the ratio method also fails to identify different photochemical production. Thus we will discuss the sources of gaseous carbonyls more detail in the next section.

Source apportionment of carbonyl compounds using observation-based model Carbonyls production at the JJS site was observed on May 17, 2018 and simulated by an OBM model (Fig. 8). The simulated distribution of $\mathrm{HCHO}$ from photochemical production was 
426 compared with the observed HCHO concentration. Peaks appeared during the day and valleys 427 appeared during the night. This was similar to the trend observed for $\mathrm{O}_{3}$, and indicated that the 428 HCHO photochemical reaction had a large contribution during the day. The highest in situ HCHO 429 was $0.64 \mathrm{ppbv} / \mathrm{h}$ at $12: 00$, and this value was lower than those measured in a previous study in 430 Beijing on July 23 and 24, 2008 (Yang et al., 2018). One aspect of the figure is of interest, two 431 large increases were observed in $\mathrm{HCHO}$ in the early morning and after dusk. Rush hour traffic 432 occurs during these two periods and vehicle emissions are a major source of $\mathrm{HCHO}$ at such times 433 (Cao et al., 2016; Tsai et al., 2014). In addition, the nocturnal boundary layer build ups rapidly 434 concentration (Brown \& Stutz, 2012).

The highest observed rate of acetaldehyde appeared at dusk, because the major source of acetaldehyde is anthropogenic emission and it accumulated during daytime. But the highest in situ photochemical rate was $0.92 \mathrm{ppbv} / \mathrm{h}$ at 11:00 due to higher photolysis rate of acetaldehyde in the afternoon. Acetone has a lower in situ photochemical rate indicated photochemical generation is the minor source of acetone. And it has a higher background value (the lowest value of acetone, about $16 \mathrm{ppbv}$ ), means it may be affected by local pollution sources on this day. Others represent the sum of other carbonyl compounds. Similar to formaldehyde, it affected by photochemical production during the daytime and anthropogenic emission during the nighttime.

At 3:00 p.m., the net $\mathrm{HCHO}$ generation rate showed a decreasing trend, indicating that the on-site $\mathrm{HCHO}$ generation rate was lower than the $\mathrm{HCHO}$ destruction rate. In fact, many simulations showed that the concentration of $\mathrm{OH}$ radicals was highest in the afternoon during the day (Tan et al., 2019; Zong et al., 2018). The photolysis of formaldehyde is an important source of $\mathrm{OH}$ radicals. Negative values occur when the rate of formaldehyde photolysis is greater than the sum of the production rate and the primary release. It has been reported in many articles that the concentration of formaldehyde decreases between 14:00 and 18:00, and according to the published literature, this phenomenon is most pronounced in summer (de Blas et al., 2019; Jiang et al., 2016; Jiang et al., 2019; Yang et al., 2019).

Fig. 9 shows that the model-calculated relative incremental reactivities (RIR) of the major precursors for secondary formation of carbonyl compounds on May 17,2018. We further identified key precursors by calculating the RIR (Cardelino \& Chameides, 1995), which has been applied in many previous studies (Tan et al., 2018; Yang et al., 2018). All VOCs species were categorized as biogenic or anthropogenic hydrocarbons (AHCs). The AHCs were divided into the following four subgroups: alkanes, alkenes, alkynes, and aromatics. All kinds of carbonyl compounds production were VOC-limited, and the dominant position was AHC. The RIRs for the NOx were negative. But the dominant species of different carbonyl compounds were different. Alkenes were dominant and aromatics followed for formaldehyde. For acetaldehyde and other carbonyl compounds, alkanes and alkenes both were important for its chemical generation, followed by aromatics. And 
466 alkanes dominate the formation of acetone. Therefore, anthropogenic VOCs emissions should be 467 reduced for carbonyl compounds controlling.

468

469

470

471

472

473

474

475

476

477

478

479

480

481

482

483

484

485

486

487

488

489

490

491

492

493

494

495

496

497

498

499

500

501

502

503

504

505

506

\section{Conclusions}

The characteristics and sources of 16 carbonyls compounds were measured in May 2018 in the southeastern coastal city of Fuzhou, China. The concentration at the urban site was $15.45 \pm 11.18$ ppbv and the concentration at the suburban site was $17.57 \pm 12.77 \mathrm{ppbv}$. HCHO, acetaldehyde, and acetone were the main species in Fuzhou city, and acetone had the highest concentration. The F/A and A/P ratios were used to determine the sources of the carbonyl compounds. Suburban areas with high vegetation coverage had high $\mathrm{F} / \mathrm{A}$ values, whereas urban areas were greatly affected by human activities and the $\mathrm{A} / \mathrm{P}$ values were higher. $\mathrm{HCHO}$ production was VOC-limited, and AHCs were dominant and particularly sensitive to reactive alkenes. The RIR for NOx were negative and NOx would generally have a negative influence on attempts to control HCHO production. In summary, both anthropogenic emission and biogenic emissions sources were important source of OVOCs in Fuzhou, and the impact of anthropogenic emission was greater, deserve more attention in the future control of ozone precursors, both in Fuzhou and other cities of China.

\section{Acknowledgements}

We sincerely thank Manfei Yin from Guizhou University, Yujie Zhang from the Chinese Research Academy of Environmental Sciences and Chenglong Zhang from the Research Center for EcoEnvironmental Sciences, Chinese Academy of Sciences for their great help. Finally, we would like to show our deep thankfulness to the reviewers and editors who have contributed valuable comments to improve the quality of the paper.

\section{References}

Atkinson R. 2000. Atmospheric chemistry of VOCs and NOx. Atmospheric Environment 34:2063-2101 DOI 10.1016/S1352-2310(99)00460-4.

Brey SJ, and Fischer EV. 2015. Smoke in the City: How Often and Where Does Smoke Impact Summertime Ozone in the United States? Environmental Science Technology 50:12881294 DOI 10.1021/acs.est.5b05218.

Brown SS, and Stutz J. 2012. Nighttime radical observations and chemistry. Chemical Society Reviews 41:6405-6447 DOI 10.1039/c2cs35181a.

Cao X, Yao Z, Shen X, Ye Y, and Jiang X. 2016. On-road emission characteristics of VOCs from light-duty gasoline vehicles in Beijing, China. Atmospheric Environment 124:146155 DOI 10.1016/j.atmosenv.2015.06.019.

Cardelino CA, and Chameides WL. 1995. An observation-based model for analyzing ozone precursor relationships in the urban atmosphere. Journal Of The Air \& Waste Management Association 45:161-180 DOI 10.1080/10473289.1995.10467356.

Crutzen PJ, and Andreae M. 1990. Biomass bu rn-ing in the tropics: Impact on atmospheric chemistry and biogeochemical cycles. Science 250:1669-1678 DOI 10.1126/science. 250.4988 .1669 . 
507

508

509

510

511

512

513

514

515

516

517

518

519

520

521

522

523

524

525

526

527

528

529

530

531

532

533

534

535

536

537

538

539

540

541

542

543

544

545

546

547

548

549

550

Dai WT, Ho SSH, Ho KF, Liu WD, Cao J, and Lee S. 2012. Seasonal and diurnal variations of mono- and di-carbonyls in Xi'an, China. Atmospheric Research 113:102-112 DOI 10.1016/j.atmosres.2012.05.001.

De Blas M, Ibanez P, Antonio Garcia J, Carmen Gomez M, Navazo M, Alonso L, Durana N, Iza J, Gangoiti G, and Estibaliz SDC. 2018. Summertime high resolution variability of atmospheric formaldehyde and non-methane volatile organic compounds in a rural background area. Science of The Total Environment 647:862-877 DOI 10.1016/j.scitotenv.2018.07.411.

Duane M, Poma B, Rembges D, Astorga C, and Larsen B. 2002. Isoprene and its degradation products as strong ozone precursors in Insubria, Northern Italy. Atmospheric Environment 36:3867-3879 DOI 10.1016/S1352-2310(02)00359-X.

Feng Y, Wen S, Chen Y, Wang X, Lu H, Bi X, Sheng G, and Fu J. 2005. Ambient levels of carbonyl compounds and their sources in Guangzhou, China. Atmospheric Environment 39:1789-1800 DOI 10.1016/j.atmosenv.2004.10.009.

Finlayson-Pitts BJ, and Pitts JN, Jr. 1997. Tropospheric air pollution: ozone, airborne toxics, polycyclic aromatic hydrocarbons, and particles. Science 276:1045-1052 DOI $10.1126 /$ science. 276.5315 .1045

He Z, Wang X, Ling Z, Zhao J, Guo H, Shao M, and Wang Z. 2019. Contributions of different anthropogenic volatile organic compound sources to ozone formation at a receptor site in the Pearl River Delta region and its policy implications. Atmospheric Chemistry and Physics 19:8801-8816 DOI 10.5194/acp-19-8801-2019.

Hong Z, Li M, Wang H, Xu L, Hong Y, Chen J, Chen J, Zhang H, Zhang Y, Wu X, Hu B, and Li M. 2019. Characteristics of atmospheric volatile organic compounds (VOCs) at a mountainous forest site and two urban sites in the southeast of China. Science Of The Total Environment 657:1491-1500 DOI 10.1016/j.scitotenv.2018.12.132.

Ho KF, Ho SSH, Huang RJ, Dai WT, Cao JJ, Tian L, and Deng WJ. 2015. Spatiotemporal distribution of carbonyl compounds in China. Environmental Pollution 197:316-324 DOI 10.1016/j.envpol.2014.11.014.

IARC, 2004. The IARC Monographs Series e IARC Classifies Formaldehyde as Carcinogenic to Humans e Press Release No. 153. World Health Organization, International Agency for Research on Cancer, Lyon, France.

IARC, 2006. IARC Monographs on the Evaluation of Carcinogenic Risks to Humans Formaldehyde, 2-Butoxyethanol and 1-tert-Butoxypropan-2-ol. World Health Organization, International Agency for Research on Cancer, Lyon, France.

Janson R, and Serves CD. 2001. Acetone and monoterpene emissions from the boreal forest in northern Europe. Atmospheric Environment 35:4629-4637 DOI 10.1016/S13522310(01)00160-1.

Jenkin ME, Saunders SM, Wagner V, and Pilling MJ. 2003. Protocol for the development of the Master Chemical Mechanism, MCM v3 (Part B): tropospheric degradation of aromatic volatile organic compounds. Atmospheric Chemistry and Physics 3:181-193 DOI 10.5194/acp-3-181-2003.

Jiang Z, Zheng X, Zhai H, Wang Y, Wang Q, and Yang Z. 2019. Seasonal and diurnal characteristics of carbonyls in the urban atmosphere of Changsha, a mountainous city in south-central China. Environmental Pollution 253:259 DOI 10.1016/j.envpol.2019.06.127.

Peer) reviewing PDF | (2020:06:50164:1:0:NEW 9 Sep 2020) 
551

552

553

554

555

556

557

558

559

560

561

562

563

564

565

566

567

568

569

570

571

572

573

574

575

576

577

578

579

580

581

582

583

584

585

586

587

588

589

590

591

592

593

594

595

596

Lü H, Cai QY, Wen S, Chi Y, Guo S, Sheng G, and Fu J. 2010. Seasonal and diurnal variations of carbonyl compounds in the urban atmosphere of Guangzhou, China. Science Of The Total Environment 408:3523-3529 DOI 10.1016/j.scitotenv.2010.05.013.

Mellouki A, Wallington TJ, and Chen J. 2015. Atmospheric chemistry of oxygenated volatile organic compounds: impacts on air quality and climate. Chemical Reviews 115:3984-4014 DOI $10.1021 /$ cr500549n.

National Climatic Data Center. Available at ftp://ftp.ncdc.noaa.gov/pub/data/noaa/isd (accessed 5 April 2019).

Pang X, and Mu Y. 2006. Seasonal and diurnal variations of carbonyl compounds in Beijing ambient air. Atmospheric Environment 40:6313-6320 DOI 10.1016/j.atmosenv.2006.05.044.

Possanzini M, Palo V, Petricca M, Fratarcangeli R, and Brocco D. 1996. Measurements of Lower Carbonyls in Rome Ambient Air. Atmospheric Environment 30:3757-3764 DOI 10.1016/1352-2310(96)00110-0.

Possanzini M, Tagliacozzo G, and Cecinato A. 2007. Ambient Levels and Sources of Lower Carbonyls at Montelibretti, Rome (Italy). Water, Air, and Soil Pollution 183:447-454 DOI 10.1007/s11270-007-9393-1.

Riemer D, Pos W, Milne P, Farmer C, Zika R, Apel E, Olszyna K, Kliendienst T, Lonneman W, Bertman S, Shepson P, and Starn T. 1998. Observations of nonmethane hydrocarbons and oxygenated volatile organic compounds at a rural site in the southeastern United States. Journal of Geophysical Research 1032:28111-28128 DOI 10.1029/98JD02677.

Saunders S, Jenkin M, Derwent R, and Pilling M. 2003. Protocol for the development of the Master Chemical Mechanism, MCM v3 (Part A): Tropospheric degradation of nonaromatic volatile organic compounds. Atmospheric Chemistry and Physics 3:161-180 DOI 10.5194/acpd-2-1847-2002.

Shepson PB, Hastie DR, Schiff HI, Polizzi M, Bottenheim JW, Anlauf K, Mackay GI, and Karecki DR. 1991. Atmospheric concentrations and temporal variations of C1 $\square$ C3 carbonyl compounds at two rural sites in central Ontario. Atmospheric Environment 25:2001-2015 DOI 10.1016/0960-1686(91)90280-K.

Sin DWM, Wong Y-C, and Louie PKK. 2001. Trends of ambient carbonyl compounds in the urban environment of Hong Kong. Atmospheric Environment 35:5961-5969 DOI 10.1016/S1352-2310(01)00359-4.

Singh HB, Kanakidou M, Crutzen PJ, and Jacob DJ. 1995. High concentrations and photochemical fate of oxygenated hydrocarbons in the global troposphere. Nature 378:5054 DOI 10.1038/378050a0.

Tago H, Kimura H, Kozawa K, and Fujie K. 2005. Formaldehyde Concentrations in Ambient Air in Urban and Rural Areas in Gunma Prefecture, Japan. Water, Air, and Soil Pollution 163:269-280 DOI 10.1007/s11270-005-0720-0.

Tan Z, Lu K, Hofzumahaus A, Fuchs H, Bohn B, Holland F, Liu Y, Rohrer F, Shao M, Sun K, Wu Y, Zeng L, Zhang Y, Zou Q, Kiendler-Scharr A, Wahner A, and Zhang Y. 2019. Experimental budgets of $\mathrm{OH}, \mathrm{HO} 2$, and $\mathrm{RO} 2$ radicals and implications for ozone formation in the Pearl River Delta in China 2014. Atmos Chem Phys 19:7129-7150 DOI 10.5194/acp-19-7129-2019.

Tan Z, Lu K, Jiang M, Su R, Dong H, Zeng L, Xie S, Tan Q, and Zhang Y. 2018. Exploring ozone pollution in Chengdu, southwestern China: A case study from radical chemistry to

Peer) reviewing PDF | (2020:06:50164:1:0:NEW 9 Sep 2020) 
599

600

601

602

603

604

605

606

607

608

609

610

611

612

613

614

615

616

617

618

619

620

621

622

623

624

625

626

627

628

629

630

631

632

633

634

635

636

637

638

639

640

641

O(3)-VOC-NO(x) sensitivity. Science Of The Total Environment 636:775-786 DOI 10.1016/j.scitotenv.2018.04.286.

The Master Chemical Mechanism VERSION 3.3.1. Available at http://mcm.leeds.ac.uk/MCM/ (accessed on 3 January 2020).

Tsai J-H, Huang P-H, and Chiang H-L. 2014. Characteristics of volatile organic compounds from motorcycle exhaust emission during real-world driving. Atmospheric Environment 99:215-226 DOI 10.1016/j.atmosenv.2014.09.076.

US EPA. 1999. Compendium Method TO-11A, Determination of Formaldehyde in Ambient Air Using Adsorbent Cartridge Followed by High Performance Liquid Chromatography (HPLC). Available at https://nepis.epa.gov/( accessed 10 February 2018)

Wang T, Xue L, Brimblecombe P, Lam YF, Li L, and Zhang L. 2017. Ozone pollution in China: A review of concentrations, meteorological influences, chemical precursors, and effects. Science Of The Total Environment 575:1582-1596 DOI 10.1016/j.scitotenv.2016.10.081.

WHO, 2000. Air Quality Guidelines for Europe. Regional Office for Europe, Copenhagen, Denmark, pp. 87-91.

Wu T, and Wang X. 2015. Emission of oxygenated volatile organic compounds (OVOCs) during the aerobic decomposition of orange wastes. Journal of Environmental Sciences-China 33:69-77 DOI 10.1016/j.jes.2015.01.006.

Xiang Z, Wang H, Stevanovic S, Jing S, Lou S, Tao S, Li L, Liu J, Yu M, and Wang L. 2017. Assessing impacts of factors on carbonyl compounds emissions produced from several typical Chinese cooking. Building and Environment 125:348-355 DOI 10.1016/j.buildenv.2017.08.045.

Xue L, Wang T, Louie PK, Luk CW, Blake DR, and Xu Z. 2014. Increasing external effects negate local efforts to control ozone air pollution: a case study of Hong Kong and implications for other Chinese cities. Environmental Science \& Technology 48:1076910775 DOI 10.1021/es503278g.

Yang X, Xue L, Wang T, Wang X, Gao J, Lee S, Blake DR, Chai F, and Wang W. 2018. Observations and Explicit Modeling of Summertime Carbonyl Formation in Beijing: Identification of Key Precursor Species and Their Impact on Atmospheric Oxidation Chemistry. Journal of Geophysical Research: Atmospheres 123:1426-1440 DOI 10.1002/2017jd027403.

Yang Z, Cheng HR, Wang ZW, Peng J, Zhu JX, Lyu XP, and Guo H. 2019. Chemical characteristics of atmospheric carbonyl compounds and source identification of formaldehyde in Wuhan, Central China. Atmospheric Research 228:95-106 DOI 10.1016/j.atmosres.2019.05.020.

Yujing, Daele, Veronique, Grosselin, Benoit, Mellouki, Abdelwahid, Jiang, and Zhaohui. 2016. Seasonal, diurnal and nocturnal variations of carbonyl compounds in the semi-urban environment of Orleans, France. Journal of environmental sciences 40:84-91 DOI 10.1016/j.jes.2015.11.016.

Zarzana KJ, Min KE, Washenfelder RA, Kaiser J, Krawiec-Thayer M, Peischl J, Neuman JA, Nowak JB, Wagner NL, Dubè WP, St Clair JM, Wolfe GM, Hanisco TF, Keutsch FN, Ryerson TB, and Brown SS. 2017. Emissions of Glyoxal and Other Carbonyl Compounds from Agricultural Biomass Burning Plumes Sampled by Aircraft. Environmental Science \& Technology 51:11761-11770 DOI 10.1021/acs.est.7b03517.

Peer) reviewing PDF | (2020:06:50164:1:0:NEW 9 Sep 2020) 
642 Zhang L, Steinmaus C, Eastmond DA, Xin XK, and Smith MT. 2009. Formaldehyde exposure 643 and leukemia: A new meta-analysis and potential mechanisms. Mutation Research/Fundamental Molecular Mechanisms of Mutagenesis 681:150-168 DOI 10.1016/j.mrrev.2008.07.002.

Zhang X, Li H, Zhang CL, et al, 2019. Optimization and Preliminary Application of the Detection Method of Carbonyl Compounds in the Ambient Air. Research of Environmental Sciences 32:821-829 DOI 10.13198/j.issn.1001-6929.2019.03.12.

Zhang Y, Xue L, Dong C, Tao W, Mellouki A, Zhang Q, and Wang W. 2019. Gaseous carbonyls in China's atmosphere: Tempo-spatial distributions, sources, photochemical formation, and impact on air quality. Atmospheric Environment 214:116863 DOI 10.1016/j.atmosenv.2019.116863.

Zhu B, Han Y, Wang C, Huang X, Xia S, Niu Y, Yin Z, and He L. 2019. Understanding primary and secondary sources of ambient oxygenated volatile organic compounds in Shenzhen utilizing photochemical age-based parameterization method. Journal of Environmental Sciences-China 75:105-114 DOI 10.1016/j.jes.2018.03.008.

Zong R, Xue L, Wang T, and Wang W. 2018. Inter-comparison of the Regional Atmospheric Chemistry Mechanism (RACM2) and Master Chemical Mechanism (MCM) on the simulation of acetaldehyde. Atmospheric Environment 186:144-149 DOI 10.1016/j.atmosenv.2018.05.013.

662 
Figure 1

Google earth map showing the location of the Fuzhou sample sites of JJS and GSS.
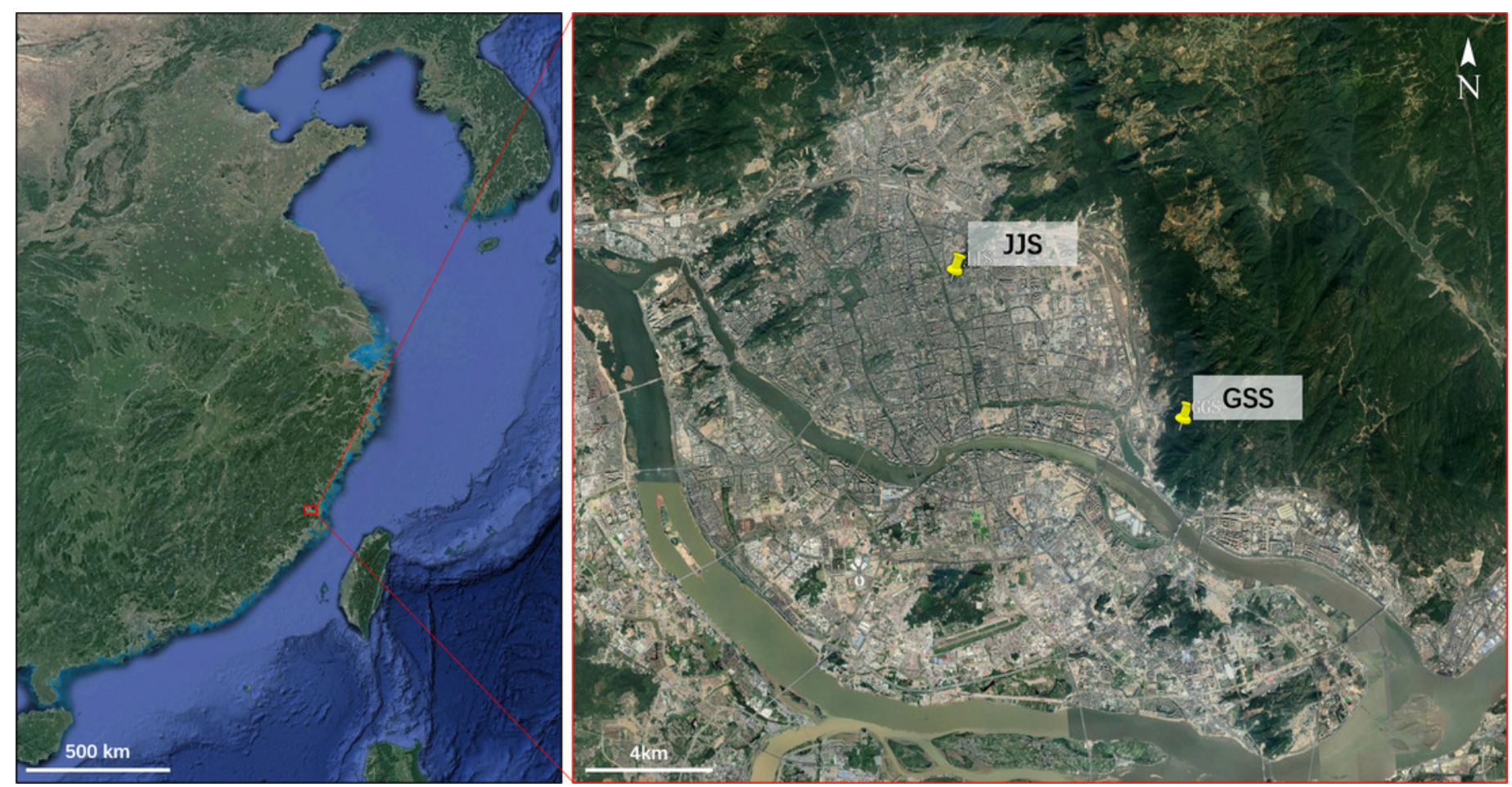
Figure 2

Time series of carbonyl compounds, $\mathrm{O}_{3}, \mathrm{NO}_{2}, \mathrm{SO}_{2}, \mathrm{CO}, \mathrm{PM}_{2.5}$, and meteorological parameters at GSS and JJS sites from May 5 to 20, 2018.

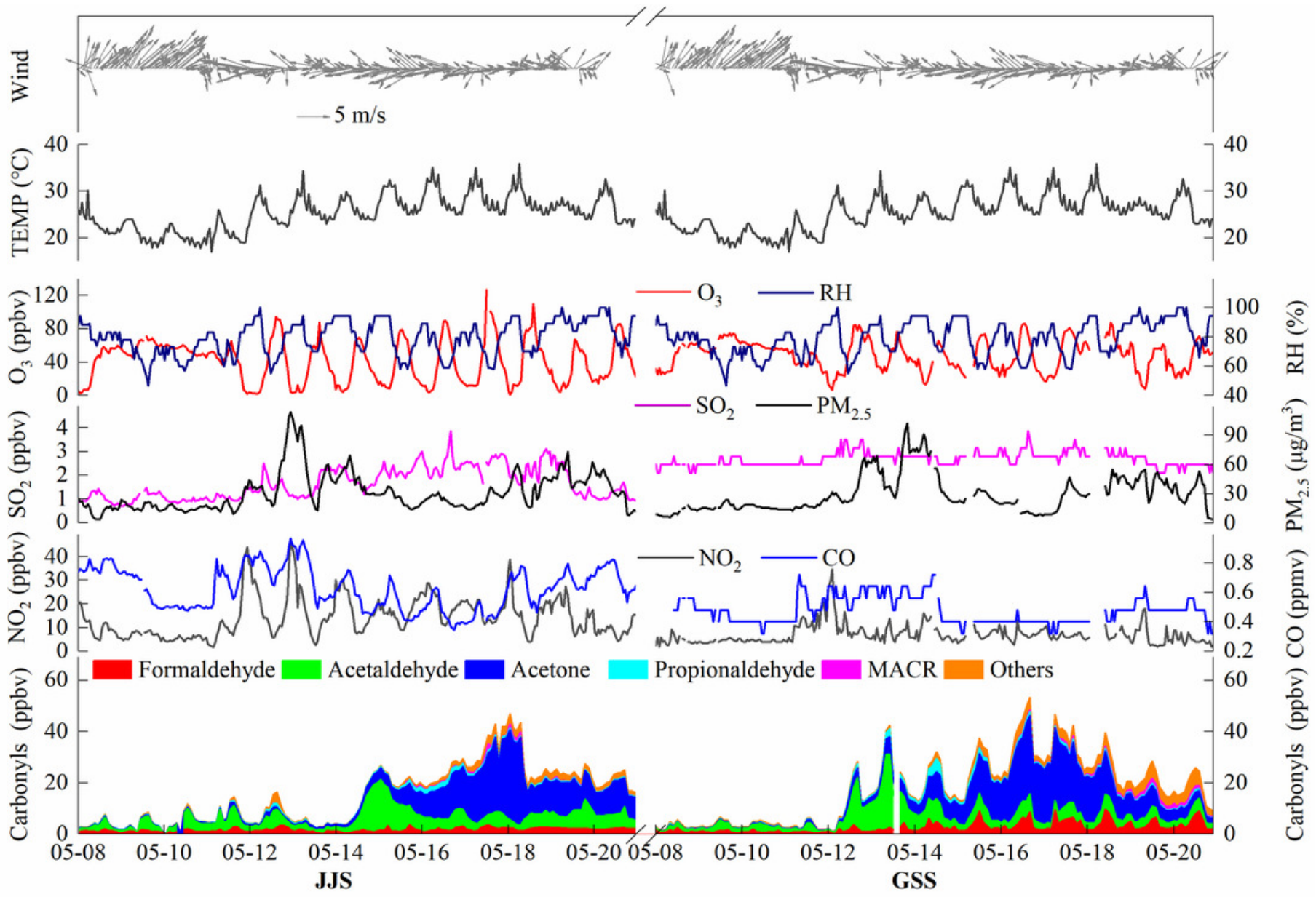


Figure 3

Diurnal variations of the total carbonyl compounds and $\mathrm{O}_{3}$ at GSS and JJS sites from May 8 and 20, 2018.
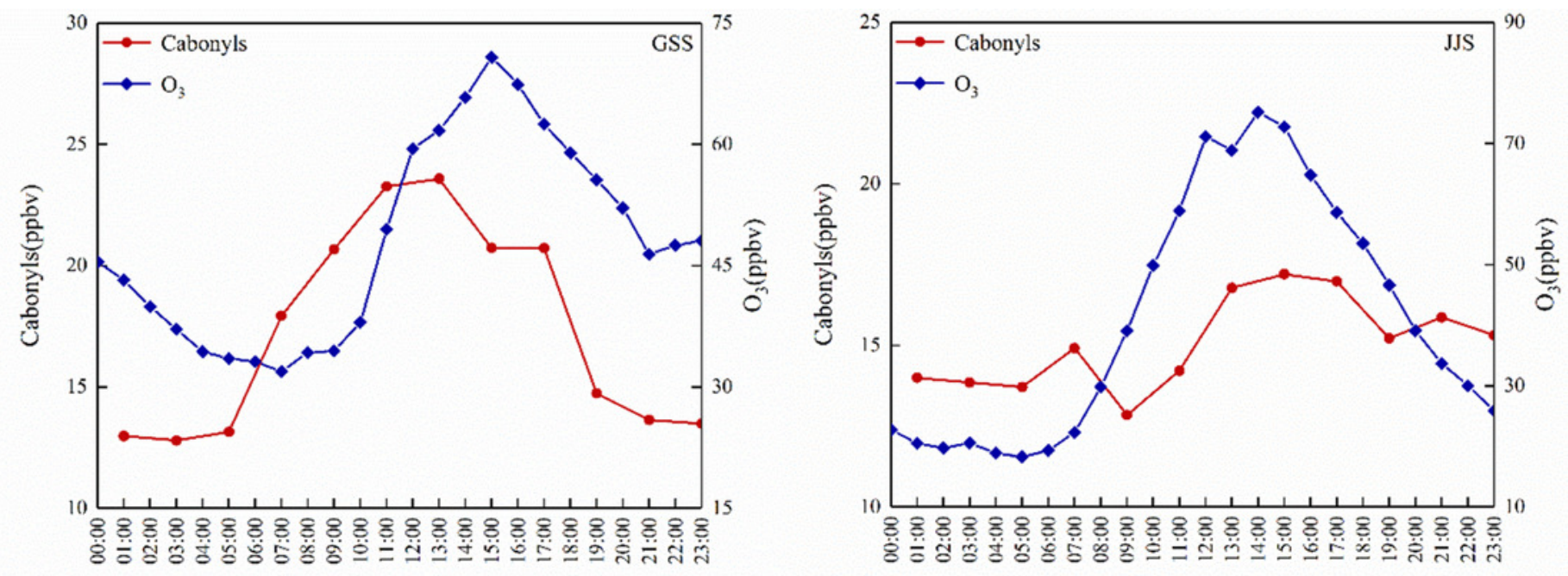
Figure 4

Diurnal variations of main carbonyl compounds at GSS and JJS sites from May 8 and 20, 2018.
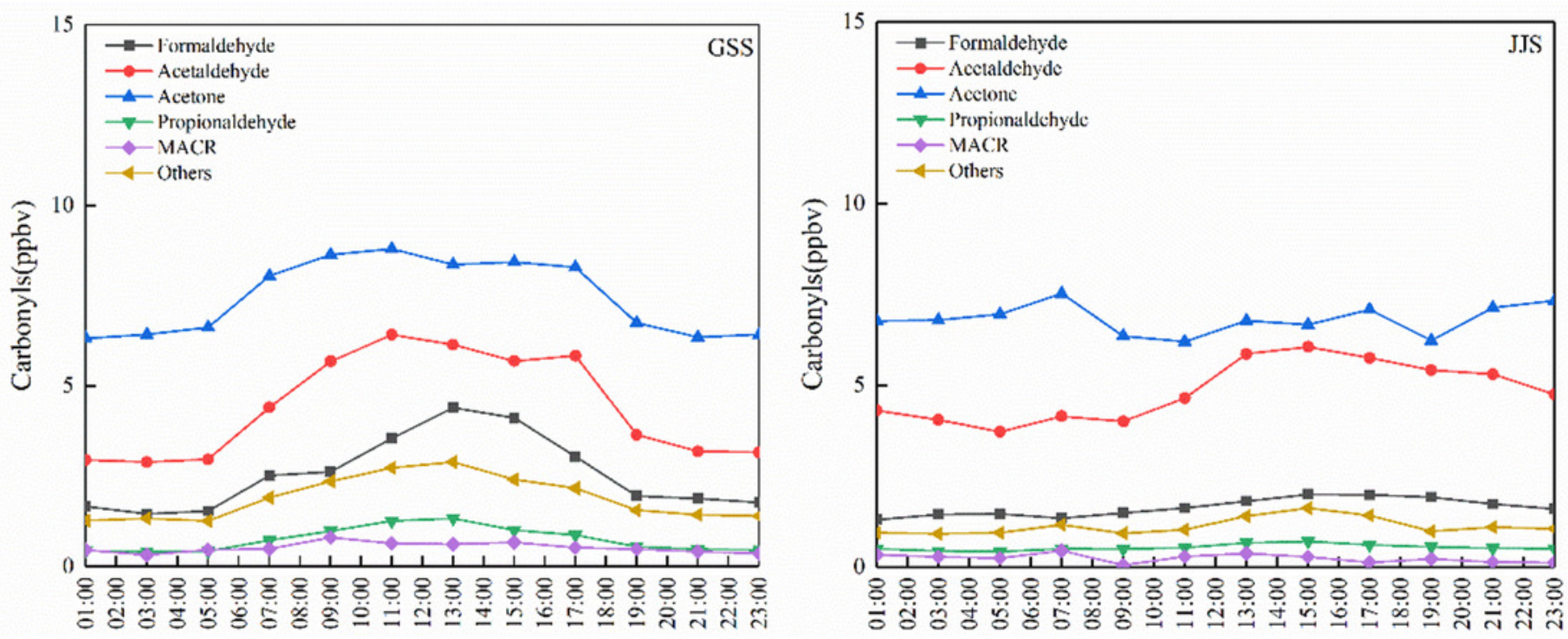


\section{Figure 5}

Proportions of main carbonyl compounds at GSS and JJS sites from May 8 to 20, 2018.

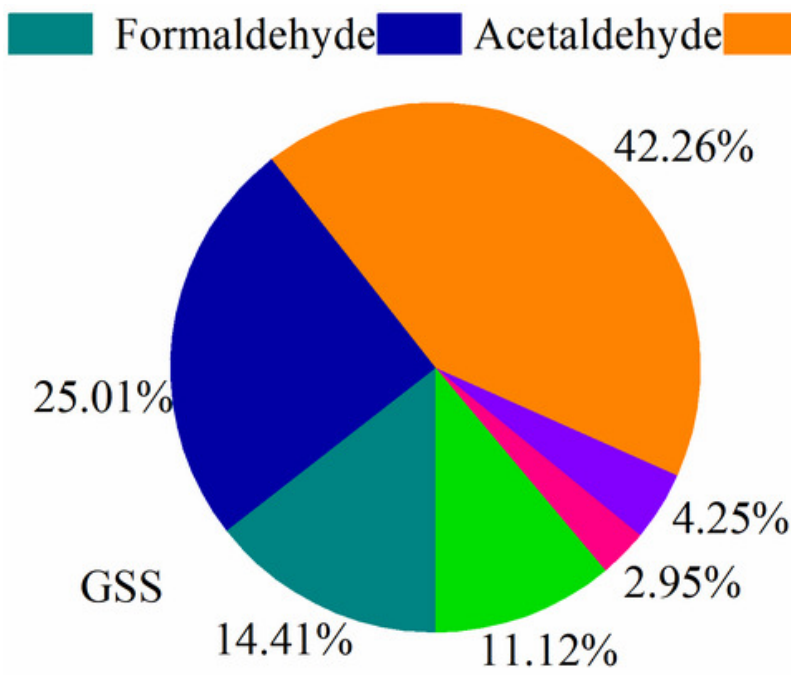

$$
\text { Acetone Propionaldehyde MACR Others }
$$

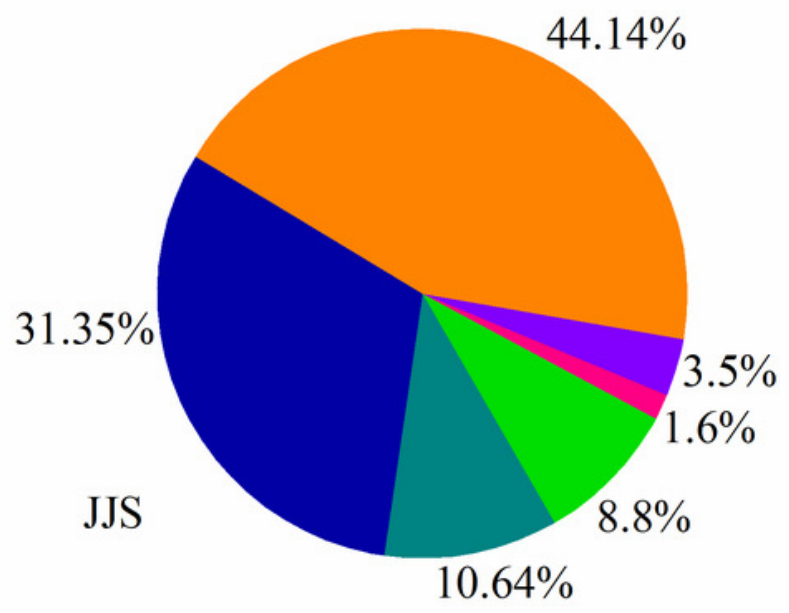


Figure 6

Ratios of formaldehyde to acetaldehyde (F/A) during the day and night at GSS and JJS sites from May 8 and 20, 2018.

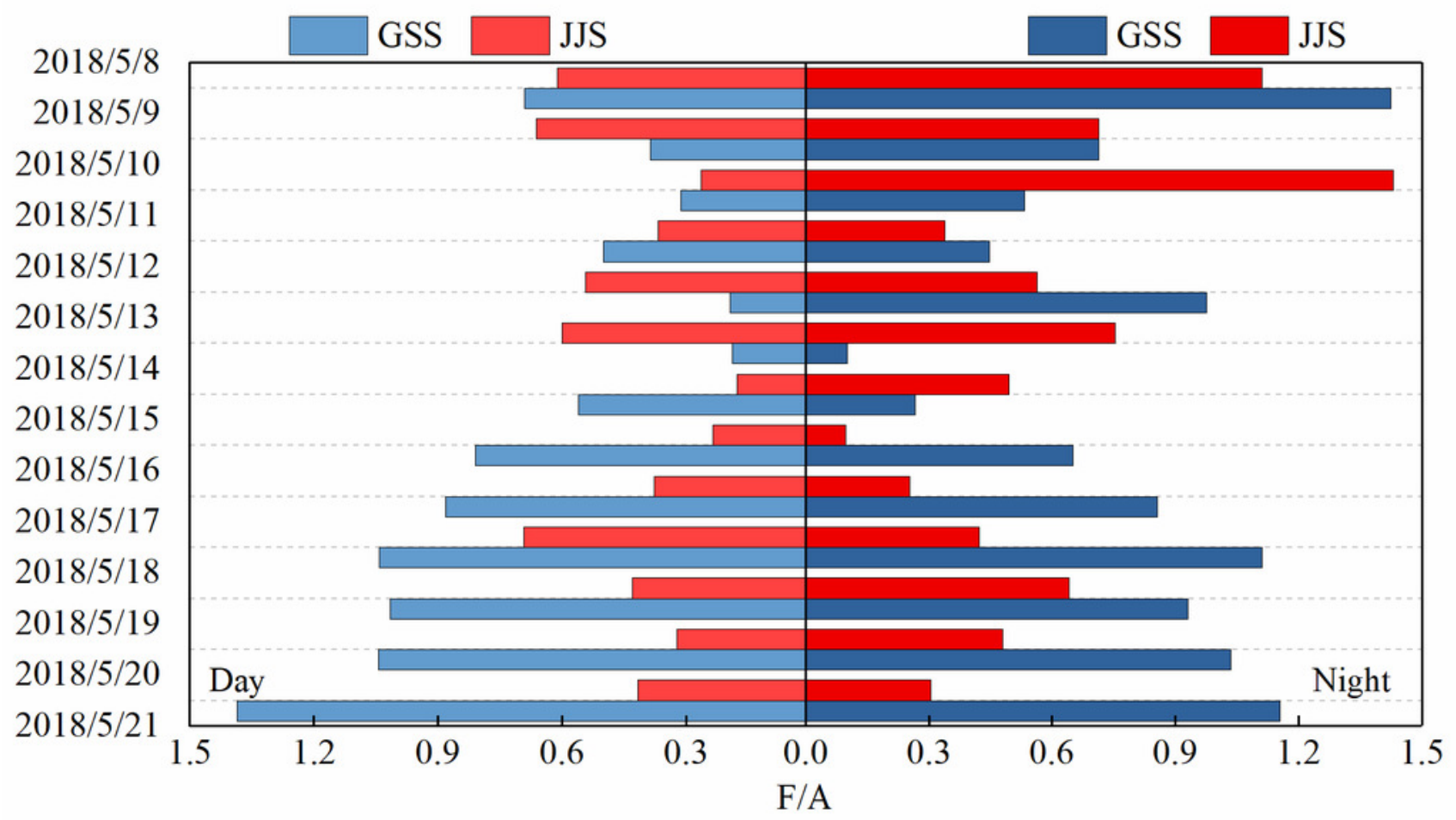


Figure 7

Ratios of acetaldehyde to propionaldehyde (A/P) during the day and night at GSS and JJS sites from May 8 and 20, 2018.

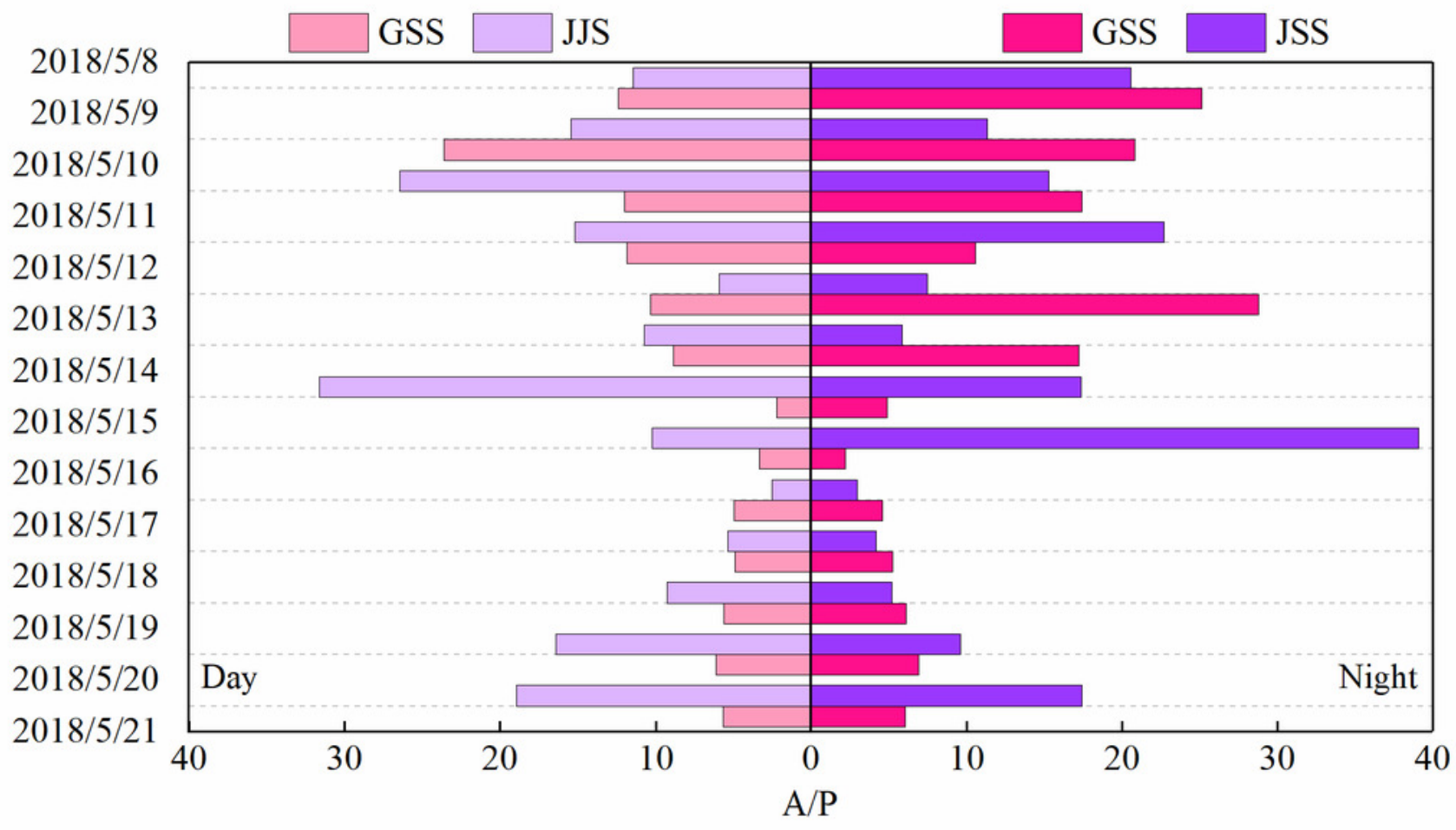


Figure 8

The simulated and observed HCHO production rate on May 17, 2018.

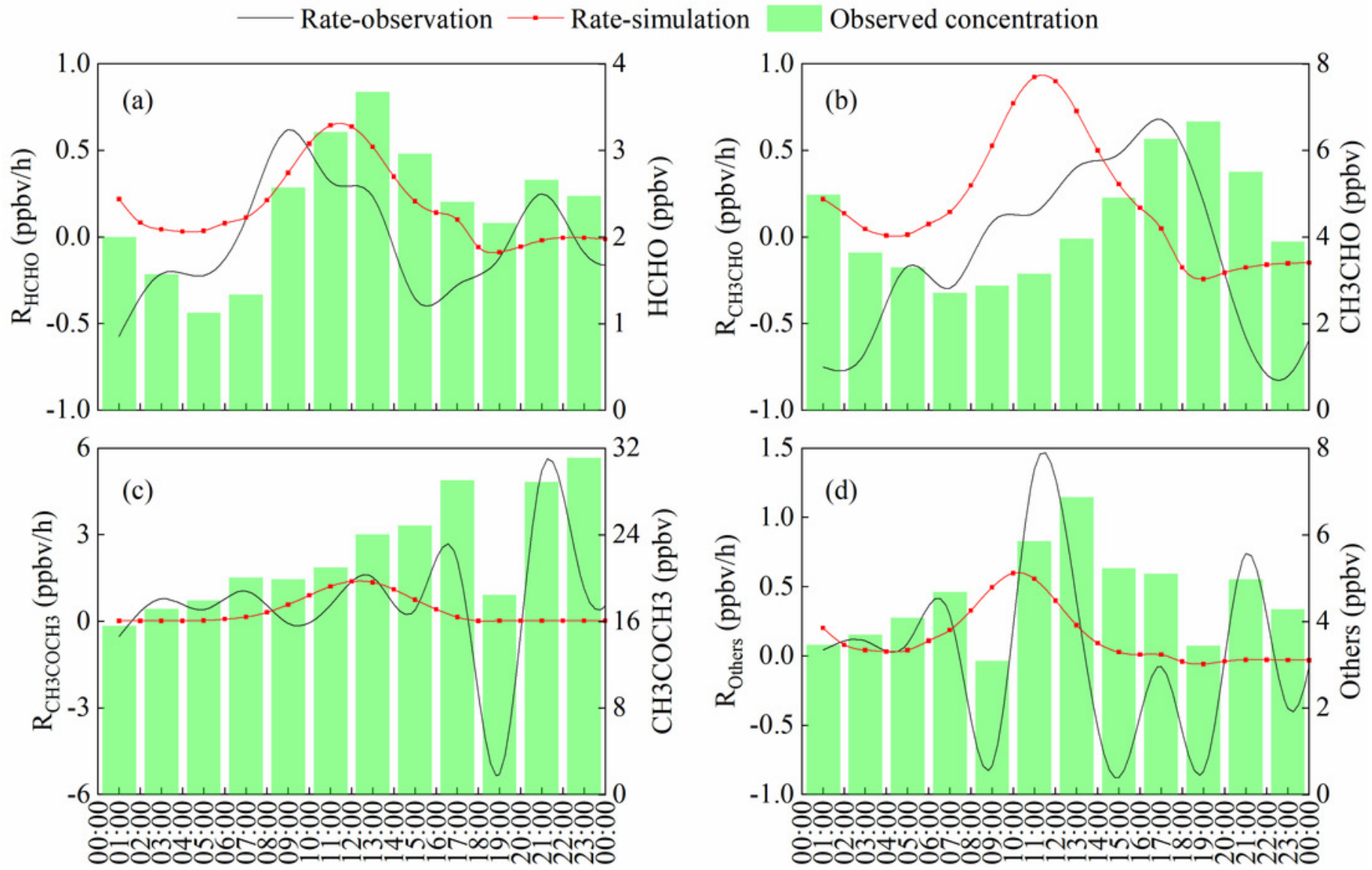


Figure 9

The model-calculated RIR of the major precursors for the secondary formation of $\mathrm{HCHO}$ on May 17, 2018.

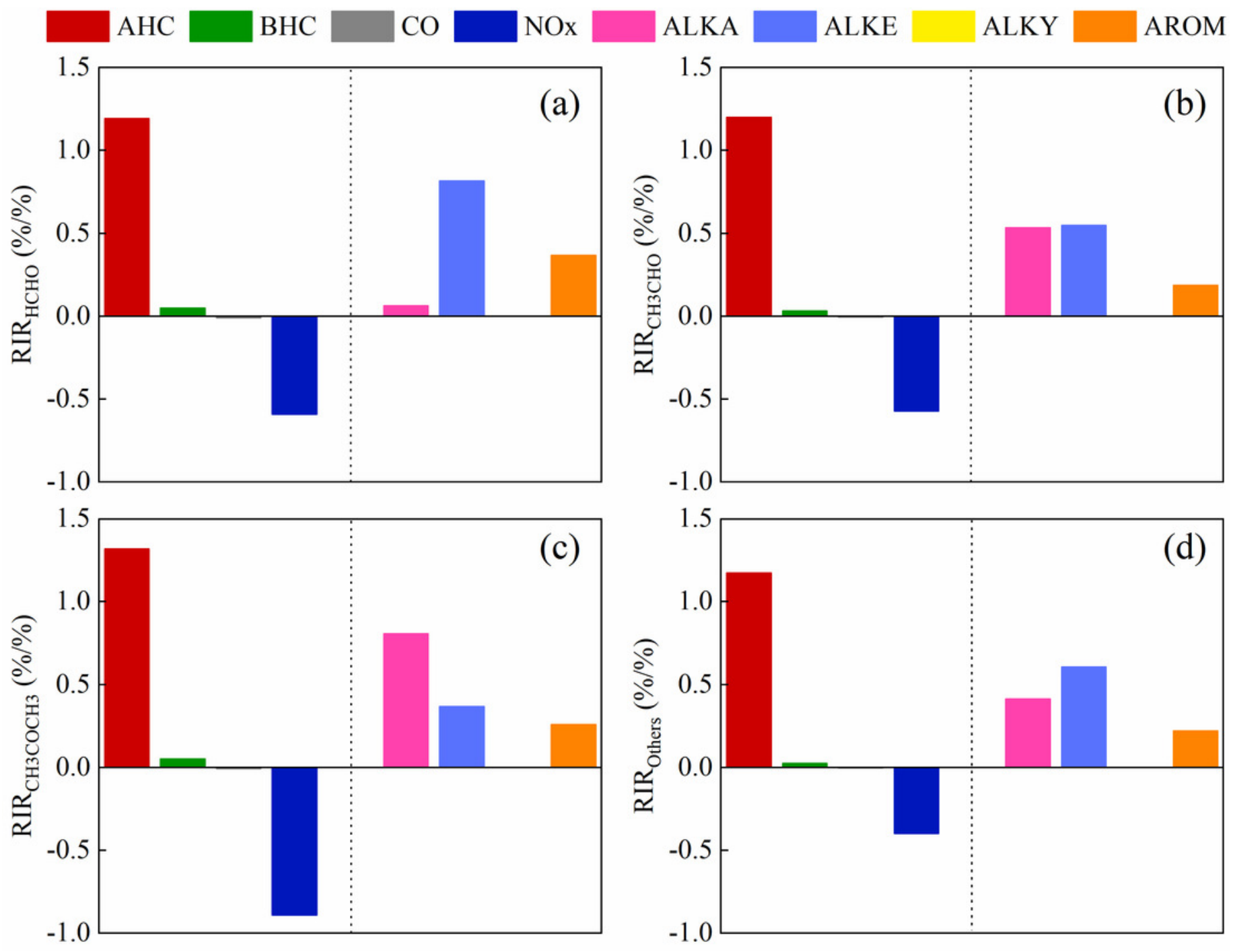


Table $\mathbf{1}$ (on next page)

Detection information of 16 carbonyl compounds by HPLC/UV. 
Table 1. Detection information of 16 carbonyl compounds by HPLC/UV.

\begin{tabular}{ccccc}
\hline Species & $\begin{array}{c}\text { Retention } \\
\text { time } \\
(\mathbf{m i n})\end{array}$ & $\begin{array}{c}\text { Correlation } \\
\text { coefficient } \\
\left(\mathbf{R}^{2}\right)\end{array}$ & $\begin{array}{c}\text { Detection } \\
\text { limit } \\
\text { (ppbv) }\end{array}$ & $\begin{array}{c}\text { Quantitation } \\
\text { limit } \\
\text { (ppbv) }\end{array}$ \\
\hline Formaldehyde & 4.97 & 1.0000 & 0.022 & 0.073 \\
Acetaldehyde & 6.24 & 1.0000 & 0.022 & 0.073 \\
Acrolein & 7.83 & 1.0000 & 0.023 & 0.076 \\
Acetone & 8.13 & 1.0000 & 0.026 & 0.085 \\
Propionaldehyde & 8.70 & 1.0000 & 0.028 & 0.092 \\
Crotoraldehyde & 10.55 & 1.0000 & 0.031 & 0.102 \\
$n$-Butyraldehyde & 12.00 & 0.9998 & 0.035 & 0.104 \\
Benzaldehyde & 13.31 & 0.9994 & 0.036 & 0.109 \\
Isovaleraldehyde & 16.20 & 0.9996 & 0.049 & 0.164 \\
Valeraldehyde & 17.13 & 0.9980 & 0.048 & 0.159 \\
$o$-Tolualdehyde & 18.32 & 0.9992 & 0.050 & 0.166 \\
$m$-Tolualdehyde & 18.95 & 0.9982 & 0.050 & 0.166 \\
$p$-Tolualdehyde & 19.76 & 0.9978 & 0.054 & 0.180 \\
Hexaldehyde & 24.21 & 0.9998 & 0.034 & 0.114 \\
2,5-Dimethylbenzaldehyde & 24.75 & 0.9998 & 0.031 & 0.102 \\
MACR & 11.67 & 0.9994 & 0.033 & 0.110 \\
\hline
\end{tabular}

2 
Table 2 (on next page)

Concentrations of $\mathrm{PM}_{2.5}, \mathrm{SO}_{2}, \mathrm{O}_{3}, \mathrm{CO}, \mathrm{NO}_{2}$ at GSS and JJS sites 
1

Table 2. Concentrations of other pollutants at GSS and JJS sites.

\begin{tabular}{ccccc}
\hline \multirow{2}{*}{ Compound } & \multicolumn{2}{c}{ Mean \pm SD $^{1}$} & \multicolumn{2}{c}{ Number of samples } \\
\cline { 2 - 5 } & GSS & JJS & GSS & JJS \\
\hline $\mathrm{PM}_{2.5}\left(\mu \mathrm{g} / \mathrm{m}^{3}\right)$ & $29,40 \pm 18.83$ & $31.06 \pm 19.36$ & $\mathrm{n}=311$ & $\mathrm{n}=311$ \\
$\mathrm{SO}_{2}(\mathrm{ppb})$ & $2.67 \pm 0.34$ & $1.65 \pm 0.66$ & $\mathrm{n}=311$ & $\mathrm{n}=311$ \\
$\mathrm{O}_{3}(\mathrm{ppb})$ & $48.29 \pm 18.03$ & $40.70 \pm 25.37$ & $\mathrm{n}=311$ & $\mathrm{n}=311$ \\
$\mathrm{CO}(\mathrm{ppm})$ & $0.47 \pm 0.09$ & $0.63 \pm 0.14$ & $\mathrm{n}=311$ & $\mathrm{n}=311$ \\
$\mathrm{NO}_{2}(\mathrm{ppb})$ & $6.99 \pm 4.15$ & $14.22 \pm 8.45$ & $\mathrm{n}=311$ & $\mathrm{n}=311$ \\
\hline
\end{tabular}

2 
Table 3 (on next page)

Concentrations of carbonyl compounds at GSS and JJS sites 
Table 3. Concentrations of carbonyl compounds at GSS and JJS sites.

\begin{tabular}{|c|c|c|c|c|}
\hline \multirow{2}{*}{ Compound } & \multicolumn{2}{|c|}{ Mean \pm SD $^{1}$ (ppbv) } & \multicolumn{2}{|c|}{ Range（ppbv） } \\
\hline & GSS & JJS & GSS & JJS \\
\hline Formaldehyde & $2.54 \pm 2.09$ & $1.64 \pm 0.75$ & $0.23-9.63$ & $0.08-3.68$ \\
\hline Acetaldehyde & $4.41 \pm 4.36$ & $4.84 \pm 3.63$ & $0.48-29.07$ & $0.02-19.61$ \\
\hline Acrolein & $\mathrm{BDL}^{2}$ & BDL & BDL & BDL \\
\hline Acetone & $7.45 \pm 8.13$ & $6.82 \pm 8.11$ & $0.03-30.55$ & BDL-35.18 \\
\hline Propionaldehyde & $0.75 \pm 0.82$ & $0.54 \pm 0.51$ & BDL-5.28 & BDL-2.20 \\
\hline Crotonaldehyde & $0.08 \pm 0.08$ & $0.07 \pm 0.07$ & BDL-0.34 & BDL-0.29 \\
\hline Butyaldehyde & $1.28 \pm 1.3$ & $0.80 \pm 0.92$ & BDL-4.57 & BDL-3.95 \\
\hline Benzaldehyde & $0.05 \pm 0.03$ & BDL & BDL-0.22 & BDL-0.15 \\
\hline Isovaleraldehyde & $0.48 \pm 0.43$ & $0.19 \pm 0.53$ & BDL-2.27 & BDL-3.71 \\
\hline Valeraldehyde & $\mathrm{BDL}$ & $\mathrm{BDL}$ & $\mathrm{BDL}$ & BDL-0.20 \\
\hline o-Tolualdehyde & $\mathrm{BDL}$ & BDL & BDL & BDL \\
\hline m-Tolualdehyde & $\mathrm{BDL}$ & BDL & BDL-0.05 & BDL-0.05 \\
\hline p-Tolualdehyde & BDL & $\mathrm{BDL}$ & $\mathrm{BDL}$ & BDL \\
\hline Hexaldehyde & $0.06 \pm 0.05$ & $0.22 \pm 0.34$ & BDL-0.36 & BDL-1.01 \\
\hline 2,5-Diemthybenzaldehyde & $\mathrm{BDL}$ & $\mathrm{BDL}$ & $\mathrm{BDL}$ & $\mathrm{BDL}$ \\
\hline MACR & $0.52 \pm 0.57$ & $0.24 \pm 0.42$ & BDL-2.01 & BDL-2.37 \\
\hline Total Carbonyl compounds & $17.57 \pm 12.77$ & $15.45 \pm 11.18$ & $1.11-53.22$ & $0.04-46.83$ \\
\hline
\end{tabular}

